\title{
Psicologia, Comportamento, Processos e Interações
}

\author{
Psychology, Behavior, Processes and Interactions
}

\author{
João Cláudio Todorov ${ }^{*}, a, b$ \& Márcio Borges Moreira ${ }^{b, c}$ \\ ${ }^{a}$ Universidade Católica de Goiás, ${ }^{b}$ Instituto de Educação Superior de Brasília \\ ${ }^{c}$ Universidade de Brasília
}

\begin{abstract}
Resumo
Em 2008 comemoram-se os 70 anos da publicação de $O$ Comportamento dos Organismos, primeiro livro de Burrhus Frederick Skinner e de análise do comportamento. Este trabalho, inspirado em uma revisão crítica do texto publicada em 1990 por Philip N. Hineline no Journal of the Experimental Analysis of Behavior, discute algumas características do livro e da análise do comportamento que tornam o comportamentalismo uma abordagem muitas vezes mal entendida por uns, assustadora para outros. $\mathrm{O}$ tema abordado é complexo e possui diversos desdobramentos. Tanto as características da obra de Skinner, como as possíveis razões para a falta de sua compreensão, serão apenas brevemente apresentadas e discutidas. O aprofundamento do tema pode ser feito percorrendo-se a literatura citada.

Palavras-chave: Análise do comportamento; O comportamento dos organismos; B. F. Skinner.

Abstract

In 2008 we celebrated the $70^{\text {th }}$ anniversary of Skinner's publication - The Behavior of Organisms. The present work is inspired by a review of the book published by Philip N. Hineline in the Journal of the Experimental Analysis of Behavior. Some characteristics of the book and of behavior analysis that make behaviorism incomprehensible for some and menacing for others are discussed. The issue discussed is complex and has many derivations. The features of Skinner's work, and possible reasons for its misunderstanding, will be only briefly presented. A more complete account of the theme may be found in the literature cited in this work.

Keywords: Behavior analysis; The behavior of organisms; B. F. Skinner.
\end{abstract}

Algumas obras fundamentais, daquelas que iniciam longos programas de pesquisa científica no sentido de Lakatos (1978), às vezes levam tempo para ser compreendidas e são os próprios resultados preliminares do programa que nos levam a perceber seu alcance. O primeiro livro de B. F. Skinner, The Behavior of Organisms (Skinner, 1938), é um desses exemplos. Grande parte dos psicólogos não percebeu esse alcance até hoje. Em 1990 Philip Hineline escreveu sobre o livro no Journal of the Experimental Analysis of Behavior (Hineline, 1990) chamando a atenção dos próprios analistas do comportamento para algumas das contribuições de Skinner que passaram despercebidas ou que demoraram a ser claramente compreendidas. Como o artigo de Hineline não parece ser muito conhecido no Brasil, vale a pena recordar seus comentários, especialmente às vésperas dos 70 anos da publicação de $O$ Comportamento dos Organismos.

O primeiro aspecto a ser notado é a sutileza da teoria, ou a maneira sutil empregada por Skinner para apresentar alguns princípios cruciais. Um exemplo claro é a

${ }^{*}$ Endereço para correspondência: SGAS Quadra 613/614, Lotes 97 e 98, Av. L-2 Sul, Campus Edson Machado, Brasília, DF, Brasil, CEP 70200-730. Tel.: (61) 34454500. E-mail: todorov@unb.br definição de reflexo, conceito pavloviano clássico (Pavlov, 1927), entendido à época como uma ligação direta estímulo-resposta: um estímulo do ambiente aciona mecanicamente uma resposta do organismo. Skinner mudou totalmente o conceito de concreto e mecânico para um conceito intrinsecamente abstrato, uma correlação entre dois eventos observáveis (Skinner, 1938). Escreveu especificamente: "Em geral, a noção de reflexo deve se livrar de qualquer noção de 'empurrão' do estímulo. Os termos se referem aqui a eventos correlacionados, e a nada mais." (Skinner, 1938, p. 21). É comum lermos ou ouvirmos que a noção de causa em análise do comportamento foi substituída pela noção de relações funcionais (e.g., Chiesa, 1994/2006; Skinner, 1953/1967). Mas o que isso de fato quer dizer? A citação anterior de Skinner (1938) sobre reflexos parece ser um bom exemplo do que significa esta substituição. Ernest Mach (cf. Chiesa, 1994/2006) gerou certa discussão entre filósofos e físicos ao afirmar que o conceito de força era absolutamente redundante para o adequado entendimento e aplicação da mecânica clássica. A noção proposta por Mach de que não é necessário inferir ou postular uma "força de atração" para explicar porque objetos caem, é a mesma noção proposta por Skinner (1938) de que não é necessário inferir uma força ou mecanismo que estabelecem o elo entre um estímulo e uma resposta. Como aponta o 
próprio Skinner (1953/1967), a ciência tem substituído o termo causa pelo termo relação funcional, pois o primeiro remete a forças e mecanismos que "ligam" dois eventos, já o segundo, apenas estabelece regularidade entre dois (ou mais) eventos.

A partir desta inovação no uso do conceito de reflexo, da própria mudança em sua definição, a teoria geral do comportamento que vem sendo desenvolvida ao longo destes 70 anos definitivamente nada tem a ver com posições mecanicistas de outras abordagens anteriores. Com a distinção entre respondentes e operantes (Skinner, 1935, 1938; Todorov, 2002), e com a substituição de tentativas discretas pelo operante livre, Skinner introduz na psicologia o tempo como variável no sentido de que o que é estudado não são eventos estáticos, pontuais, mas processos de interação que ocorrem no tempo (Todorov, 1985), processos de interação entre comportamento, não organismo, e ambiente. Contudo, vários dos livros-texto de introdução ou de história da psicologia e renomados pensadores (e.g., Capra, 1983; Chomsky, 1959; Eysenck, 1984; Schultz \& Schultz, 1969/1992) insistem em caracterizar a contribuição de Skinner como mecanicista, $\mathrm{S}-\mathrm{R}$, estímulo-resposta, exatamente as posições às quais Skinner estava se contrapondo. Ignorância ou má fé?

Chiesa (1994/2006) fornece uma revisão extensa, porém não exaustiva sobre esse assunto, mas não é preciso ir muito longe, no tempo, para encontrar parte da resposta. Já em O Comportamento dos Organismos, logo nas primeiras páginas, Skinner (1938) sinaliza que a contingência S-R (estímulo-resposta) diz respeito apenas à parte do comportamento do organismo, notadamente o comportamento reflexo, e introduz a noção de comportamento operante, distinguindo, inicialmente, reflexos eliciados de comportamentos emitidos. Tal ampliação e os princípios comportamentais dela derivados, ainda que incipientes em 1938, parecem não terem sido percebidas ou compreendidas por alguns críticos de Skinner. Aliás, compreensão talvez não seja um termo adequado, dada a clareza de algumas afirmações encontradas no livro, por exemplo: "Uma emoção é um processo dinâmico, em vez de uma relação estática entre um estímulo e uma resposta." (1938, p. 409).

Moore (1984) também fornece alguns exemplos interessantes de referências equivocadas à obra de Skinner, comentando algumas possíveis origens desses equívocos. Moore argumenta que tais equívocos podem ser categorizados, genericamente, em cinco tipos principais e mais recorrentes:

... algumas concepções equivocados sobre o behaviorismo radical têm sido prevalentes nos livros-texto de psicologia, como documentado por Todd e Morris (1983). Em resumo, essas concepções equivocadas envolvem o seguinte: (a) que está preocupada primariamente com o comportamento de animais nãohumanos, (b) que é totalmente ambientalista, (c) que expõe uma abordagem do 'organismo vazio' ou da 'caixa preta',(d) que expõe uma teoria simples e ingê- nua sobre a linguagem e aquisição da linguagem, e (e) que é limitada e sem utilidade. (Moore, 1984, p. 392-393).

Outra contribuição do livro (Skinner, 1938) costuma ser deturpada, ainda que sobejamente expandida e esclarecida pelos livros que se seguiram: Ciência e Comportamento Humano (Skinner, 1953/1967), Comportamento Verbal (Skinner, 1957/1978), Contingências de Reforçamento (Skinner, 1969/1984), Sobre o Behaviorismo (Skinner, 1974/1982), e, entre outros, o que teve o título errada e horrivelmente traduzido para " $O$ Mito da Liberdade" (Skinner, 1971/1983). Trata-se da crítica às explicações mentalistas, que costumam ser tomadas como negação da mente. Para Skinner, então, segundo seus críticos, o organismo seria uma caixa vazia, recebendo o empurrão de estímulos e reagindo com respostas automáticas. Essa deturpação seria cômica se não fosse trágica, ao revelar a completa falta de compreensão da proposta de Skinner por vários autores - muitos deles seus críticos. A título de ilustração, examinemos as seguintes palavras de William Baum:

O termo mentalismo foi adotado por B. F. Skinner para se referir a um tipo de "explicação" que na verdade não explica nada. Suponha que você pergunte a um amigo por que ele comprou um par de sapatos e a resposta seja "Comprei porque quis", ou "Comprei por impulso". Embora essas afirmações soem como explicações, você na verdade não avançou nada em relação a sua pergunta. Essas não explicações são exemplos de mentalismo. (Baum, 1994/1999, p. 47).

Como fica claro no trecho citado acima (Baum, 1994/ 1999), dizer que uma explicação é mentalista não é equivalente a falar de um "organismo vazio", mas a mera especificação de um erro lógico na atribuição de causalidade a fenômenos comportamentais: o comportamento observado é, na explicação mentalista, a única evidência do "processo mental que o causou", gerando um raciocínio tautológico que se estende ad infinitum. Há uma diferença dramática entre dizer que uma emoção não é causa de um outro comportamento e dizer que o indivíduo não tem emoções (ou pensamentos, ou lembranças, etc.). No entanto, via de regra, quando o analista do comportamento destitui de certos processos comportamentais (ou psíquicos) o status de causa da ação, o não-analista do comportamento insiste em acreditar que se destitui o organismo tais "faculdades mentais".

Um claro exemplo dessa confusão entre status de causa versus ignorar um fenômeno pode ser encontrada em Aronson, Wilson e Akert (1999/2002), um importante livro introdutório de psicologia social. Além da confusão em relação ao status de causa (e talvez em função dela), os autores parecem ignorar também a vasta contribuição da análise do comportamento para a compreensão de fenômenos sociais, relatada, entre outras fontes, em um periódico específico chamado Behavior and Social Issues: 
Os behavioristas escolheram não tratar de assuntos como cognição, pensamento e sentimento, porque consideravam conceitos vagos e mentalistas demais e não-ancorados o suficiente no comportamento observável. Elegante em sua simplicidade, a abordagem behaviorista pode explicar uma grande faixa de comportamentos. Mas, como não inclui cognição, pensamentos e sentimentos - fenômenos esses vitais à experiência social humana -, essa abordagem revelou-se inadequada para a plena compreensão do mundo social. (Aronson, Wilson, \& Akert, 1999/2002, p. 9).

$\mathrm{O}$ problema aqui parece ser de dificuldade de mudar a maneira de pensar característica da chamada sociedade ocidental, a civilização como desenvolvida a partir dos gregos e de seus filósofos. Atribuir a eventos mentais, internos, ou a presumíveis processos fisiológicos do organismo a causa do comportamento que se observa parece tão natural que é assim que domina o senso comum. Uma excelente constatação do quanto este modo de pensar é característico da sociedade ocidental é dada por Bennett e Hacker (2003). Os autores fazem uma revisão do uso de conceitos psicológicos utilizados por neurocientistas, apontando a recorrência daquilo que chamam de falácia mereológica, que consiste basicamente na atribuição de predicados ao cérebro ou parte dele que, de acordo com a lógica do uso dos conceitos psicológicos, só podem ser atribuídos ao todo, ao organismo. Bennett e Hacker (2003) mostram como o dualismo mente-corpo transformou-se no dualismo cérebro/neurônio-corpo.

Uma teoria que não contraria o senso comum tem muito mais chances de adquirir adeptos que uma que nos faz repensar o mundo. A análise do comportamento é uma das poucas abordagens na psicologia que escapa da classificação tradicional de estudo de interações organismocomportamento. Alguns exemplos de teorias que enfatizam eventos no organismo como causas de comportamentos observáveis são a sociobiologia, a psicologia cognitiva, a psicologia evolutiva, a psicobiologia, a psicanálise e a psicologia humanista. Entre as abordagens que enfatizam as relações ambiente-comportamento estão a análise do comportamento, a teoria dos sistemas, e a abordagem sócio-histórica (Todorov, 1989).

A discussão sobre se a análise do comportamento é ou não é antimentalista se perde quando não se percebe essa diferença fundamental em relação à maioria das outras abordagens focadas na interação organismo-comportamento. A análise do comportamento vê o que está atrás dos olhos como eventos a serem explicados para então ser parte da explicação. Estuda-se a relação ambientecomportamento sabendo que parte da ação está ocorrendo atrás dos olhos, embaixo dos cabelos e entre as duas orelhas; às vezes, também só acima do céu da boca. Mas considera também que outra parte da explicação está no ambiente imediato e em várias outras interações anteriores ambiente-comportamento que ocorreram no passado próximo ou remoto.
Ainda que se pudesse pensar na associação desses dois tipos de teoria em um tipo mais geral, na prática e na história o que ocorre é bem diferente: elas geram experimentos diferentes, dão mais força explicativa a causas diferentes. Discordam quanto ao status do comportamento observável. As teorias baseadas no organismo olham para o comportamento como indício ou sintoma de processos que ocorrem dentro do organismo, sejam eles presumíveis processos fisiológicos ou processos mentais metafóricos (a mente processando informações como um computador, por exemplo). Já em uma teoria baseada no ambiente o comportamento é o foco principal e a teoria se fundamenta em relações ambiente-comportamento. Veja-se, por exemplo, o tratamento dado à subjetividade na abordagem sócio-histórica, totalmente compatível com a abordagem skinneriana da questão, e incompatível com abordagens centradas no organismo (Hayes, Hayes, \& Reese, 1988; Hineline, 1990; Morris, 1988; Todorov, 1989). Por outro lado, Maria Malott demonstra a compatibilidade da análise do comportamento com a teoria dos sistemas ao trabalhar teórica e praticamente com organizações complexas (Glenn \& Malott, 2004; Malott, 2003; Martone \& Todorov, 2005; Todorov, Martone, \& Moreira, 2006) e Steven Hayes (2004) amplia o alcance da teoria ao enfatizar o contextualismo (Todorov, 1989) e o estabelecimento de relações bidirecionais estímulo-estímulo, gerando novos procedimentos educacionais e terapêuticos, entre outros.

A ênfase na interação ambiente-comportamento, e não em processos hipotéticos dos quais o comportamento seria apenas um subproduto, ou um mero sintoma, permitiu que a análise do comportamento se tornasse uma das poucas abordagens em Psicologia - quiçá a única - que, a partir de um mesmo referencial teórico-metodológico, consegue lidar com qualquer fenômeno comportamental/ psicológico. Analistas do comportamento têm atuado com sucesso na clínica (com todos os transtornos psicológicos), nas organizações, nas escolas, nos esportes, nos hospitais, com bebês, crianças, jovens, adultos, idosos, com pessoas ditas normais ou com algum atraso no desenvolvimento. Duas obras recentes, publicadas em português, podem ser um bom começo para o leitor interessado em conhecer as contribuições da análise do comportamento: Análise do Comportamento para a Educação: Contribuições Recentes (Hübner \& Marinott, 2004) e Análise do Comportamento: Pesquisa, Teoria e Aplicação (Abreu-Rodrigues \& Ribeiro, 2005).

A "dificuldade de compreensão" da obra de Skinner, e de seus sucessores, mesmo 70 anos depois da publicação de O Comportamento dos Organismos (Skinner, 1938), é tamanha que se inicia já no entendimento de dois conceitos básicos: comportamento e ambiente. Quando nos referimos a fenômenos comportamentais, ou comportamentos, parece ainda necessário esclarecer que estamos no referindo a pensamentos (e.g., Donahoe \& Palmer, 1994; Skinner, 1953/1967, 1974/1982), emoções (e.g., 
Banaco, 1993; Friman, Hayes \& Wilson, 1998; Skinner, 1953/1967, 1974/1982), linguagem (e.g., Catania, 1998/ 1999; Sidman, 1994; Skinner, 1957/1978), religiosidade (e.g., Banaco, 2001; Rachlin, 2007; Schoenfeld, 1982; Skinner, 1953/1967), subjetividade (e.g., Costa, 2002; Skinner, 1953/1967, 1974/1982), livre-arbítrio (e.g., Chiesa, 1994/2006; Skinner, 1971/1983), cognição (e.g., Catania, 1998/1999; Donahoe \& Palmer, 1994; Palmer, 2003; Sidman, 1994; Simonassi, 1999), memória (e.g., Catania, 1998/1999; Donahoe \& Palmer, 1994; Oliveira-Castro, 1992), sentimentos (e.g., Friman et al., 1998; Skinner, 1989/1991), auto-conhecimento (e.g., Skinner, 1953/1967, 1974/1982; Tourinho, 1994), autocontrole (e.g., Hanna \& Todorov, 2002; Rachlin, 1974; Skinner, 1953/1967), personalidade (e.g., Moreira, 2007; Skinner, 1974/1982), criatividade (e.g., Barbosa, 2003; Skinner, 1968), motivação (e.g., Michael, 1982; Skinner, 1953/ 1967; Todorov \& Moreira, 2005), cultura (e.g., Glenn, 2003; Guerin, 1994; Lamal, 1991, 1997; Malott \& Glenn, 2006; Sidman, 1989/1995; Skinner, 1953/1967; Todorov, Martone \& Moreira, 2005), política e leis (e.g., Goldstein \& Pennypacker, 1998; Skinner, 1953/1967; Todorov, 2005; Todorov, Moreira, Prudêncio, \& Pereira, 2004), problemas sociais (e.g., Biglan, 1995; Mattaini \& Thyer, 1996; Skinner, 1953/1967; Todorov \& Moreira, 2004), entre outros. Da mesma forma, o conceito de ambiente estende-se muito além do espaço físico. Uma noção adequada de ambiente, sob a ótica da análise do comportamento, pode ser encontrada em Todorov (1989).

Um raro, porém dramático reconhecimento do impacto da análise do comportamento feito por um não-analista do comportamento foi dado pelo psicólogo cognitivista Roediger (2004/2005), então presidente da American Psychological Society (APS):

... (está tudo bem com os debates sobre a Teoria da Mente no autismo, mas não se o que você deseja é terapia e tratamento; neste caso, procure o behaviorismo) ... Num sentido muito real, todos os psicólogos contemporâneos - pelo menos aqueles que conduzem pesquisas empíricas - são behavioristas. Mesmo os experimentalistas de máxima orientação cognitiva estão estudando algum tipo de comportamento. Eles podem estar estudando os efeitos de variáveis relacionadas com o apertar de teclas de computadores, de preencher checklists, de completar avaliações de confiabilidade, de padrões de fluxo sanguíneo ou de lembrar palavras escrevendo-as em folhas de papel, mas eles estão estudando, na maioria absoluta das vezes, comportamentos objetivamente verificáveis. (Até mesmo experiências subjetivas, tais como avaliações de confiabilidade, podem ser replicadas entre diferentes pessoas e em diferentes condições). Este passo, o de passar a estudar comportamentos objetivamente verificáveis, representa uma enorme mudança em relação ao trabalho que muitos psicólogos conduziam em 1904. (p. 4-5).
Roediger (2004/2005) acrescenta ainda que onde quer que previsão e controle do comportamento sejam importantes, lá estarão analistas do comportamento trabalhando.

Outra diferença fundamental que a análise do comportamento deve ao seu livro-mãe está na linguagem de termos técnicos empregada por Skinner (1938), ainda que se possa lamentar que tenha tomado de empréstimo vários termos pavlovianos e com isso ter gerado muita confusão para quem começa a aprender a teoria. A maioria das abordagens da psicologia usa a linguagem do cotidiano, do dia a dia, aquilo que as pessoas usam quando falam com as outras, ou quando escrevem para as outras. Além disso, têm explicações semelhantes às do senso comum quanto à temporalidade, quanto à proximidade no tempo entre uma causa e um efeito (algo que a espécie humana parece compartilhar com outras espécies animais). Com frequiência se esquivam de questões como livre-arbítrio e com isso não ferem a susceptibilidade do mundo ocidental. Mas esse é um problema antigo. Em Guerra e Paz Tolstói já comentava:

Como na astronomia a dificuldade de reconhecer a moção da Terra reside no abandono da sensação imediata da estabilidade da terra e a percepção do movimento dos planetas, na história a dificuldade de reconhecer a sujeição da personalidade às leis do espaço, tempo e causa reside na renúncia do sentimento direto da independência da personalidade de cada pessoa. Mas na astronomia a nova visão disse: 'É verdade que não sentimos o movimento da Terra, mas ao admitir sua imobilidade chegamos ao absurdo, enquanto que ao admitir sua moção (a qual não sentimos) chegamos às leis', assim na história a nova visão diz: 'É verdade que não somos conscientes de nossa dependência, mas ao admitir nosso livre arbítrio chegamos ao absurdo, enquanto que ao admitir nossa dependência do mundo externo, quanto a tempo e a causa, chegamos a leis' . (Tolstoi, 1865/1952. Trecho transcrito em Catania \& Hineline, 1996, p. 592).

É contra esse pano de fundo conservador, que parece ignorar as revoluções provocadas por Darwin e por Freud, que a análise do comportamento estuda todo e qualquer comportamento, inclusive os chamados de livre escolha das pessoas, à luz de interações ambiente-comportamento, e com linguagem técnica característica da ciência (e.g., Neuringer, Jensen, \& Piff, 2007). A vantagem do uso de termos técnicos está no sentido único que carregam, sua definição e nada mais. Uma palavra como atribuição, um termo usado na Teoria da Atri-buição (Heider, 1958; Ross, 1977), tem vários outros sentidos na linguagem cotidiana, o que sempre levanta a possibilidade de confusão na comunicação. Boa parte do treino do analista do comportamento consiste em aprender a usar de forma bastante específica termos como resposta, comportamento, ambiente, reforço, etc. 
Quem ainda não percebeu que Freud acabou com o mito do homem racional (muitos economistas ainda não perceberam), e ainda mais, aqueles outros que ainda não perceberam que Darwin nos mostrou como somos mais um dos animais, realmente não estão com suas mentes greco-romanas preparadas para enfrentar o desafio do século 21: usar os métodos da ciência para aprender como planejar a sustentabilidade deste imenso condomínio (Biglan, 1995; Rakos, 1992), desafio este já abordado por Skinner (1953/1967) quase seis décadas atrás.

Recentemente um aluno do primeiro autor apresentou uma monografia requerida por um concurso de seleção de candidatos a um emprego. Skinner era vastamente citado em seu trabalho. Uma das entrevistadoras da banca de seleção estranhou as citações e perguntou: "Por que você lê tanto Skinner? Skinner é perigoso!". Hineline já tinha percebido esse efeito que às vezes causamos:

Tanto os psicólogos sociais quanto as pessoas leigas estão propensos a reagir às interpretações do analista do comportamento como insensíveis a uma dimensão muito importante na textura do discurso social. Em seus termos técnicos próprios, as interpretações dos analistas do comportamento são vistas como falhas de controle de estímulos. Assim como uma pessoa reage com legítima apreensão à "salada de palavras" que caracteriza a fala esquizofrênica sem ser capaz de discriminar o que é estranho ou ameaçador naquela fala, essa pessoa também pode agir como se estivesse sendo ameaçada pela interpretação skinneriana. (Hineline, 1990, p. 312) ${ }^{1}$.

Para Hineline, ainda que o primeiro livro de Skinner tenha desenvolvido os princípios e os conceitos que vieram a se desenvolver no que hoje é a análise do comportamento (e.g.,_Andery \& Sério, 1999; Glenn, 1988, 1991, 2004; Glenn, Ellis, \& Greenspoon, 1992; Guerin, 1992; Lamal, 1997; Malott \& Glenn, 2006; Mattaini \& Thyer, 1996; Pierce, 1991; Rakos, 1992, 1993; Todorov, 1982, 1985, 1987, 1991, 2001, 2004), aquela contribuição poderia ter sido relegada não fosse o trabalho de formação e divulgação feito por Fred S. Keller e William N. Schoenfeld na Universidade de Columbia, Nova Iorque, com a introdução do curso de laboratório de psicologia experimental e com seu livro "Princípios de Psicologia" (Keller \& Schoenfeld, 1950/1966), um livro extremamente didático e bem mais "user friendly" que o de Skinner $(1938)^{2}$.

Por fim, gostaríamos de ressaltar dois importantes aspectos relativos ao estudo comportamento que parecem,

\footnotetext{
${ }^{1} \mathrm{O}$ medo do novo (neofobia) e do diferente também parece ser algo que partilhamos com várias outras espécies animais (Cavigelli \& McClintock, 2003; Greenberg, 1983).

${ }^{2}$ A contribuição de Fred S. Keller para o desenvolvimento da análise do comportamento no Brasil pode ser vista, entre outras fontes, em Bori (1996), Gorayeb (1996), Guilhardi e Madi (1996), Keller (1968, 1972, 1974, 1975, 1982, 1987a, 1987b, 1996a, 1996b, 1996c) e Todorov $(1990,1996,2003,2006)$
}

também, dificultar de alguma maneira a compreensão das proposições básicas feitas por Skinner em 1938. Como dissemos anteriormente, segundo a proposta de Skinner, a Psicologia deveria estudar interações. Entretanto, o caráter processual da mudança de comportamento, bem como a intermitência e sutileza dos aspectos ambientais que influenciam o comportamento podem, de certo forma, facilitar a crença de que o comportamento dos organismos está mais sob o controle de variáveis internas ao próprio organismo do que de uma história de interações com seu ambiente. Para ilustrar esses pontos, apresentamos a seguir a tradução de um trecho do livro de Isadore Tversky, A Maimonides Reader, que ilustra razoavelmente bem estes dois pontos.

. . . Imagine uma criança pequena que foi levada a seu professor para aprender o Torah, para seu bem porque a levará ao caminho da perfeição. Contudo, porque ela é apenas uma criança e porque seu tirocínio é deficiente, ela não percebe o verdadeiro valor daquele bem, nem entende a perfeição que pode ser atingida por meio do Torah. É necessário, portanto, que o professor, que adquiriu perfeição maior que a da criança, a suborne para que estude por meio de coisa que a criança ama de uma maneira infantil. Assim, o professor pode dizer, 'Leia e eu lhe darei algumas nozes e uns figos; vou te dar um pouco de mel'. Com essa estimulação a criança tenta ler. Ela não trabalha com afinco por causa da leitura em si, pois não entende o seu valor. Ela lê para obter a comida . . . À medida que a criança cresce e sua mente se aperfeiçoa, o que antigamente era importante para ela perde sua importância, enquanto outras coisa tornam-se preciosas. A partir daí o professor vai estimular o interesse da criança seja pelo que for. O professor pode dizer, 'Leia e te darei sapatos bonitos e lindas roupas'. Assim a criança vai se dedicar à leitura para ganhar roupas e não pelo estudo a em si . . . À medida que sua inteligência melhora ainda mais e essas coisas, também, tornam-se desimportantes para a criança, seu interesse volta-se para coisas de maior valor. Então o professor pode dizer para ele, 'Leia esta passagem do capítulo que eu te dou um dinar ou dois'. Outra vez ela tentará ler para receber o dinheiro, pois dinheiro é mais importante para ela do que o estudo. O que ela quer conseguir com seu estudo é o dinheiro que lhe foi prometido. Quando seu juízo já tiver se desenvolvido mais plenamente de forma que mesmo o dinheiro já não lhe pareça atraente, ela vai desejar algo mais honroso. Seu professor pode lhe dizer, então, 'Estude para que você possa se tornar o presidente de um tribunal, um juiz, e assim as pessoas vão respeitá-lo, levantar-se ante sua presença, como fazem com Fulano de Tal'. O aluno vai então se esforçar mais ainda para alcançar esse objetivo. Vai trabalhar para chegar às honras, à exaltação e ao elogio que os outros podem lhe dar. Bem, tudo isso é deplorável. Contudo, 
é inevitável pela visão limitada do homem, que faz com que ele tome como objetivo da sabedoria outras coisas que não a própria sabedoria e supõe que o propósito de estudar é obter honrarias, o que faz da verdade um objeto de zombaria. Nossos sábios chamaram isso de aprendizagem espúria ... As massas . . . não perdem nada quando obedecem aos dez mandamentos por medo de punição e pela esperança de recompensa, pois não são perfeitos. É bom para eles na medida em que os reforça e habitua naquilo que o Torah requer. Por esse esforço eles podem ser despertos para o conhecimento da verdade e vir a servir a Deus por amor. É isso que os sábios queriam dizer quando disseram, 'Um homem deve sempre trabalhar no Torah, mesmo que não seja apenas pelo Torah! Fazendo isso ele pode vir a fazer isso apenas pelo Torah'. (Tversky, 1972, p. 404-407).

A análise do comportamento, como toda e qualquer ciência natural, não se propõe a "inventar" novos princípios. Sua tarefa é descrever regularidades existentes no mundo (e que provavelmente existem desde que o mundo é mundo), especificamente nas interações comportamento-ambiente, formulando leis científicas que nos ajudem a entender melhor o mundo, prever certos eventos e alterar a probabilidade de ocorrência de alguns deles.

A citação anterior (Tversky, 1972) ilustra alguns pontos importantes sobre os princípios comportamentais descritos por Skinner já em 1938 e sobre a má compreensão ou rejeição de tais princípios. O primeiro deles refere-se ao fato de que mudança de comportamento ou dito de maneira mais ampla, mudança de atitude - é, na maioria das vezes, um processo, e não algo pontual (Catania, 1998/1999). É difícil até mesmo para o mais convicto dos analistas do comportamento identificar mudanças no comportamento de um indivíduo observando poucas interações desse indivíduo com seu ambiente natural. Tal dificuldade é ainda mais marcante quando o observador em questão não tem uma longa história de observação do comportamento em situações controladas - o laboratório.

Outro aspecto importante retratado na citação de Tversky (1972) refere-se ao uso de reforçadores arbitrários e/ou condicionados no controle do comportamento (Keller \& Schoenfeld, 1950/1966). Recentemente um programa televisivo apresentou uma reportagem que ilustra bem a dificuldade da maioria das pessoas de entender o uso de tais reforçadores e, consequentemente, a efetividade e aplicabilidade de alguns dos princípios comportamentais descritos por Skinner (1938, 1953/ 1967). Discutia-se na reportagem se pais devem ou não "pagar salários" a seus filhos por cumprirem suas "tarefas de filhos" (estudar, ajudar em tarefas domésticas, etc.). Alguns pais diziam na reportagem que não se pode "comprar os filhos", eles devem "compreender" que estudar, por exemplo, é importante para eles e eles deveriam estudar por esse motivo, e não para serem remunerados.
Como apontado na citação de Tversky (1972), não é desejável que uma criança estude apenas para receber certa quantia de dinheiro ou brinquedos, mas sim que se comportamento de estudar seja mantidos pelos reforçadores naturais, condicionados ou não, que este comportamento pode produzir (Keller \& Schoenfeld, 1950/ 1966). Entretanto, como a mudança de comportamento é um processo (Catania, 1998/1999), muitas vezes longo; como o comportamento não precisa ser reforçado todas as vezes que ocorre para continuar ocorrendo (Ferster \& Skinner, 1957); como nem sempre é fácil identificar a ocorrência de reforçadores naturais; como muitas vezes se esquece o poder reforço social (Skinner, 1953/ 1967), como uma simples vocalização, um "umhum", por exemplo (Greenspoon, 1955); como muitas vezes não se programa contingências adequadas para que o comportamento deixe de ficar, gradualmente, sob o controle do reforçador arbitrário, e passe a ficar sob o controle de reforçadores naturais; e como o efeito do reforço condicionado é subjetivo, dependente de variáveis particulares da vida de cada indivíduo, não devemos realmente esperar que alguém compreenda facilmente a proposta de Skinner.

No entanto, como descrito anteriormente, mesmo que de forma sucinta, a ampla aplicabilidade e eficácia do uso dos princípios comportamentais encontrados na obra de Skinner, e daqueles que o sucederam, valem o esforço de tentar entendê-la, valem o esforço de deixar, pelo menos temporariamente, possíveis preconceitos de lado e tentar analisá-la com outros olhos.

\section{Referências}

Abreu-Rodrigues, J., \& Ribeiro, M. R. (Eds.). (2005). Análise do comportamento: Pesquisa, teoria e aplicação. Porto Alegre, RS: Artmed.

Andery, M. A., \& Sério, T. M. (1999). O conceito de metacontingência: Afinal, a velha contingência de reforçamento é suficiente? In R. A. Banaco (Ed.), Sobre comportamento e cognição: Aspectos teóricos, metodológicos e de formação em análise do comportamento e terapia cognitivista (2. ed., pp. 106-116). Santo André, SP: ARBytes.

Aronson, E., Wilson, T. D., \& Akert, R. M. (2002). Psicologia social (3. ed.). Rio de Janeiro, RJ: LTC. (Original publicado em 1999)

Banaco, R. A. (1993). Emoções e ação pedagógica na infância: Contribuições da psicologia comportamental. Temas em Psicologia, 1, 57-65.

Banaco, R. A. (2001). Religião e psicoterapia. Fragmentos de Cultura, 11, 55-64.

Barbosa, J. I. C. (2003). A criatividade sob o enfoque da análise do comportamento. Revista Brasileira de Terapia Comportamental e Cognitiva, 5, 185-193.

Baum, W. M. (1999). Compreender o behaviorismo: Ciência, comportamento e cultura. Porto Alegre, RS: Artmed. (Original publicado em 1994)

Bennett, M. R., \& Hacker. P. M. S. (2003). Philosophical foundations of neuroscience. Malden, MA: Blackwell. 
Biglan, A. (1995). Changing cultural practices: A contextualist framework for intervention research. Reno, NV: Context Press.

Bori, C. M. (1996). Chapters in the life of Fred S. Keller. Psicologia: Teoria e Pesquisa, 12, 189-190.

Catania, A. C. (1999). Aprendizagem: Comportamento, linguagem e cognição (4. ed.). Porto Alegre, RS: Artmed. (Original publicado em 1998)

Catania, A. C., \& Hineline, P. N. (Ed.). (1996). Variations and selections: An anthology of reviews from The Journal of the Experimental Analysis of Behavior. Bloomington, IN: SEAB.

Capra, F. (1983). The turning point: Science, society and the rising culture. London: Fontana.

Cavigelli, S. A., \& McClintock, M. K. (2003). Fear of novelty in infant rats predicts adult corticosterone dynamics and an early death. Proceedings of the National Academy of Sciences, 100, 16131-16136.

Chiesa, M. (2006). Behaviorismo radical: A filosofia e a ciência. Brasília, DF: IBAC. (Original publicado em 1994)

Chomsky, N. (1959). Verbal behavior, by B. F. Skinner. Language, 35, 26-58.

Costa, N. (2002). Terapia analítico-comportamental: Dos fundamentos filosóficos à relação com o modelo cognitivista. Santo André, SP: ESETec.

Donahoe, J. W., \& Palmer, D. C. (1994). Learning and complex behavior. London: Allyn and Bacon.

Eysenck, M. A. (1984). Handbook of cognitive psychology. London: Lawrence Erlbaum.

Ferster, C. B., \& Skinner, B. F. (1957). Schedules of reinforcement. New York: Appleton-Century-Crofts.

Friman, P. C., Hayes, S. C., \& Wilson, K. G. (1998). Why behavior analysts should study emotion: The example of anxiety. Journal of Applied Behavior Analysis, 31, 137-156.

Greenberg, R. (1983). The role of neophobia in determining the degree of foraging specialization in some migrant warblers. American Naturalist, 122, 444-453.

Glenn, S. S. (1988). Contingencies and metacontingencies: Toward a synthesis of behavior analysis and cultural materialism. The Behavior Analyst, 11, 161-169.

Glenn, S. S. (1991). Contingencies and metacontingencies: Relations among behavioral, cultural, and biological evolution. In P. A. Lamal (Ed.), Behavioral analysis of societies and cultural practices (pp. 39-73). New York: Hemisphere.

Glenn, S. S. (2003). Operant contingencies and the origin of cultures. In K. A. Lattal \& P. N. Chase (Eds.), Behavior theory and philosophy (pp. 223-242). New York: Klewer Academic.

Glenn, S. S. (2004). Individual behavior, culture, and social change. The Behavior Analyst, 27, 133-151.

Glenn, S. S., Ellis, J., \& Greenspoon, J. (1992). On the revolutionary nature of the operant as a unit of behavioral selection. American Psychologist, 47, 1329-1336.

Glenn, S. S., \& Malott, M. E. (2004). Complexity and selection: Implications for organizational change. Behavior and Social Issues, 13, 89-106.

Goldstein, M. K., \& Pennypacker, H. S. (1998). From candidate to criminal: The contingencies of corruption in elected public office. Behavior and Social Issues, 8, 1-8.

Gorayeb, R. (1996). Introdução ao texto de Fred Keller "Imagens da vida de um professor". Psicologia: Teoria e Pesquisa, 12, 3-4.
Greenspoon, J. (1955). The reinforcing effect of two spoken sounds on the frequency of members of two verbal response classes. American Journal of Psychology, 68, 409-416.

Guerin, B. (1992). Behavior analysis and the social construction of knowledge. American Psychologist, 47, 1423-1432.

Guerin, B. (1994). Analyzing social behavior: Behavior analysis and the social sciences. Reno, NV: Context Press.

Guilhardi, H., \& Madi, M. B. B. P. (1996). Professor Keller disse sim... Psicologia: Teoria e Pesquisa, 12, 113-114.

Hanna, E. S., \& Todorov, J. C. (2002). Modelos de autocontrole na análise experimental do comportamento: Utilidade e crítica. Psicologia: Teoria e Pesquisa, 18, 337-343.

Hayes, S. C. (2004). Contextualism. In W. E. Craighead \& C. B. Nemeroff (Eds.), The concise Corsini encyclopedia of psychology and behavioral science (3. ed., pp. 222-223). New York: Wiley.

Hayes, S. C., Hayes, L. J., \& Reese, H. W. (1988). Finding the philosophical core: A review of Stephen C. Pepper's World Hypotheses: A study in evidence. Journal of the Experimental Analysis of Behavior, 50, 97-111.

Heider, F. (1958). The psychology of interpersonal relations. New York: Wiley.

Hineline, P. N. (1990). The origins of environment-based psychological theory. Journal of the Experimental Analysis of Behavior, 53, 305-320.

Hübner, M. M. C., \& Marinott, M. (Eds.). (2004). Análise do comportamento para a educação: Contribuições recentes. Santo André, SP: ESETec.

Keller, F. S. (1968). Good bye teacher... Journal of Applied Behavior Analysis, 1, 79-89.

Keller, F. S. (1972). Adeus, mestre! Ciência e Cultura, 24, 207-212.

Keller, F. S. (1974). Ten years of personalized instruction. Teaching of Psychology, 1, 4-9.

Keller, F. S. (1975). On my experience in Brazil. Boletim de Psicologia, 26, 105-110.

Keller, F. S. (1982). Pedagogue's progress. Lawrence, KA: TRI.

Keller, F. S. (1987a). Itens de um fichário. Psicologia: Teoria e Pesquisa, 3, 84-91.

Keller, F. S. (1987b). O nascer de um departamento. Psicologia: Teoria e Pesquisa, 3, 198-205.

Keller, F. S. (1996a). Imagens da vida de um professor. Psicologia: Teoria e Pesquisa, 12, 5-10.

Keller, F. S. (1996b). What happened to the Brasilia Plan in the United States? Psicologia: Teoria e Pesquisa, 12, 115-119.

Keller, F. S. (1996c). Report on the Brasilia Plan. Psicologia: Teoria e Pesquisa, 12, 193-197.

Keller, F. S., \& Schoenfeld, W. N. (1966). Princípios de psicologia. São Paulo, SP: EPU. (Original publicado em 1950)

Lakatos, I. (1978). Mathematics, science and epistemology. In Philosophical papers: Vol. 2. Cambridge, MA: Cambridge University Press.

Lamal, P. A. (1991). Behavioral analysis of societies and cultural practices. New York: Hemisphere.

Lamal, P. A. (1997). Cultural contingencies: Behavior analytic perspectives on cultural practices. Westport, CT: Praeger.

Malott, M. E. (2003). Paradox of organizational change: Engineering organizations with behavioral systems analysis. Reno, NV: Context Press.

Malott, M. E., \& Glenn, S. S. (2006). Targets of intervention in cultural and behavioral change. Behavior and Social Issues, 15, 31-56. 
Todorov, J. C. \& Moreira, M. B. (2009). Psicologia, Comportamento, Processos e Interações.

Martone, R. C., \& Todorov, J. C. (2005). Complexidade e seleção: Implicações para mudança organizacional. Revista Brasileira de Terapia Comportamental e Cognitiva, 7, 197-203.

Mattaini, M. A., \& Thyer, B. A. (1996). Finding solutions to social problems: Behavioral strategies for change. Washington, DC: American Psychological Association.

Michael, J. (1982). Distinguishing between discriminative and motivational functions of stimuli. Journal of Experimental Analysis of Behavior, 37,149-155.

Moore, J. (1984). On the tactful specification of meaning: A review of Harré an Lamb's The encyclopedic dictionary of psychology. Journal of the Experimental Analysis of Behavior, 41, 387-395.

Moreira, M. B. (2007). Curtindo a vida adoidado: Personalidade e causalidade no behaviorismo radical. In A. K. C. R. De-Farias \& M. R. Ribeiro (Eds.), Skinner vai ao cinema (pp. 11-29). Santo André, SP: ESETec.

Morris, E. K. (1988). Contextualism: The world view of behavior analysis. Journal of Experimental Child Psychology, 46, 289-323.

Neuringer, A., Jensen, G., \& Piff, P. (2007). Stochastic matching and the voluntary nature of choice. Journal of Experimental Analysis of Behavior, 88, 1-28.

Oliveira-Castro, J. M. (1992). Fazer na cabeça: Uso metafórico e negativo. Psicologia: Teoria e Pesquisa, 8, 267-272.

Palmer, D. C. (2003). Cognition. In K. A. Lattal \& P. N. Chase (Eds.), Behavior theory and philosophy (pp. 167-185). New York: Kluwer Academic.

Pavlov, I. P. (1927). Conditioned reflexes: An investigation of the physiological activity of the cerebral cortex. London: Oxford University Press.

Pierce, W. D. (1991). Culture and society: The role of behavioral analysis. In P. A. Lamal (Ed.), Behavioral analysis of societies and cultural practices (pp. 13-37). New York: Hemisphere.

Rakos, R. (1992). Achieving the just society in the 21st century: What can Skinner contribute? American Psychologist, 47, 1499-1506.

Rakos, R. (1993). Propaganda as stimulus control: The case of Iraq invasion of Kuwait. Behavior and Social Issues, 3 , 35-62.

Rachlin, H. (1974). Self-control. Behaviorism, 2, 94-107.

Rachlin, H. (2007). Cui bono? A review of breaking the spell: Religion as a natural phenomenon by Daniel C. Dennett. Journal of the Experimental Analysis of Behavior, 87, 143149.

Roediger, H. L. (2005). O que aconteceu com o behaviorismo. Revista Brasileira de Análise do Comportamento (Brasília), 1, 1-6. (Original publicado em 2004)

Ross, L. (1977). The intuitive psychologist and his shortcomings: Distortions in the attribution process. In $\mathrm{L}$. Berkowitz (Ed.), Advances in experimental social psychology (Vol. 10, pp. 173-220). New York: Academic Press.

Schoenfeld, W. N. (1982). Religion and human behavior. Boston: Authors Cooperative.

Schultz, D. P., \& Schultz, S. E. (1992). História da psicologia moderna (5. ed.). São Paulo, SP: Cultrix. (Original publicado em 1969)

Sidman, M. (1994). Equivalence relations and behavior: A research story. Boston: Authors Cooperative.
Sidman, M. (1995). Coerção e suas implicações. Campinas, SP: Psy. (Original publicado em 1989)

Simonassi, L. E. (1999). Cognição: Contato com contingências e regras. Revista Brasileira de Terapia Comportamental e Cognitiva (Campinas), 1, 83-93.

Skinner, B. F. (1935). The generic nature of the concepts of stimulus and response. Journal of General Psychology, 12, 40-65.

Skinner, B. F. (1938). The behavior of organisms. New York Appleton-Century.

Skinner, B. F. (1967). Ciência e comportamento humano. Brasília, DF: Editora da Universidade de Brasília. (Original publicado em 1953)

Skinner, B. F. (1968). The creative student. In B. F. Skinner, The technology of teaching (pp. 169-184). New York: Appleton.

Skinner, B. F. (1984). Contingências do reforço: Uma análise teórica. In Os pensadores (Vol. 51, pp. 158-396). São Paulo, SP: Abril Cultural. (Original publicado em 1969)

Skinner, B. F. (1982). Sobre o behaviorismo. São Paulo, SP Cultrix. (Original publicado 1974)

Skinner, B. F. (1978). O comportamento verbal. São Paulo, SP: Cultrix. (Original publicado em 1957)

Skinner, B. F. (1983). O mito da liberdade. São Paulo, SP Summus. (Original publicado em 1971)

Skinner, B. F. (1991). Questões recentes na análise comportamental. Campinas, SP: Papyrus. (Original publicado em 1989)

Todorov, J. C. (1982). Behaviorismo e análise experimental do comportamento. Cadernos de Análise do Comportamento, $3,10-23$.

Todorov, J. C. (1985). O conceito de contingência tríplice na análise do comportamento humano. Psicologia: Teoria e. Pesquisa, 1, 140-146.

Todorov, J. C. (1987). A constituição como metacontingência. Psicologia: Ciência e Profissão, 7, 9-13.

Todorov, J. C. (1989). A psicologia como o estudo de interações. Psicologia: Teoria e Pesquisa, 5, 325-347.

Todorov, J. C. (1990). The K \& S in Brazil. Journal of the Experimental Analysis of Behavior, 54, 151-152.

Todorov, J. C. (1991). O conceito de contingência na psicologia experimental. Psicologia: Teoria e Pesquisa, 7, 59-70.

Todorov, J. C. (1996). Goodbye teacher, good old friend. Journal of the Experimental Analysis of Behavior, 66, 7-9.

Todorov, J. C. (2001). Quem tem medo de punição? Revista Brasileira de Terapia Comportamental e Cognitiva, 3, 3740.

Todorov, J. C. (2002). A evolução do conceito de operante Psicologia: Teoria e Pesquisa, 18, 123-127.

Todorov, J. C. (2003). The translation of Science and Human Behavior into Portuguese: Ciência e comportamento humano. Journal of the Experimental Analysis of Behavior, 80, 341-343.

Todorov, J. C. (2004). Da aplysia à constituição: Evolução de conceitos na análise do comportamento. Psicologia: Reflexão e Crítica, 17, 151-156.

Todorov, J. C. (2005). Laws and the complex control of behavior. Behavior and Social Issues, 14, 86-90.

Todorov, J. C. (2006). Behavior analysis in Brazil. Avances en Psicología Latinoamericana, 24, 29-36.

Todorov, J. C., Martone, R. C., \& Moreira, M. B. (Eds.). (2005). Metacontingências: Comportamento, cultura e sociedade Santo André, SP: ESETec. 
Todorov, J. C., Martone, R. C., \& Moreira, M. B. (2006). Novas diretrizes curriculares, metacontingências e o Plano Keller. In H. J. Guilhardi \& N. C. Aguirre (Eds.), Sobre comportamento e cognição: Expondo a variabilidade (Vol. 18, pp. 456-464). Santo André, SP: ESETec.

Todorov, J. C., \& Moreira, M. (2004). Análise experimental do comportamento e sociedade: Um novo foco de estudo. Psicologia: Reflexão e Crítica, 17, 25-29.

Todorov, J. C., \& Moreira, M. B. (2005). O conceito de motivação na psicologia. Revista Brasileira de Terapia Comportamental e Cognitiva, 7, 119-132.

Todorov, J. C., Moreira, M., Prudêncio, M. R. A., \& Pereira, G. C. C. (2004). O Estatuto da Criança e do Adolescente como metacontingência. In M. Z. S. Brandão, F. C. S. Conte, F. S. Brandão, Y. K. Ingberman, V. L. M. Silva, \& S. M. Oliani (Eds.), Sobre comportamento e cognição: Contingências e metacontingências, contextos sócio-verbais e o comportamento do terapeuta (Vol. 13, pp. 44-51). Santo André, SP: ESETec.

Tourinho, E. Z. (1994). A noção pragmatista de conhecimento e a noção skinneriana de conhecimento de si mesmo. Acta Comportamentalia, 2, 219-232.

Tversky, I. (1972). A Maimonides reader. Cambridge, MA: Harvard University Press. 